\title{
Uso de los valores de hormona paratiroidea intranodular obtenidos por punción con aguja fina como factor predictivo de hipoparatiroidismo posoperatorio
}

\author{
Use of intranodular parathyroid hormone values obtained by fine needle aspiration as a \\ predictive factor of postoperative hypoparathyroidism
}

\author{
Ana A. Tejera-Hernández ${ }^{1}$, Fructuoso Rodríguez-Rodríguez ${ }^{1}, M^{a}$ Isabel Gutiérrez-Giner ${ }^{1}$, \\ Yaiza López-Plasencia², Francisco J. Alcalá-Serrano ${ }^{1}$ y Juan R. Hernández-Hernández' \\ ${ }^{1}$ Servicio de Cirugía General y del Aparato Digestivo; ${ }^{2}$ Servicio de Endocrinología y Nutrición. Hospital Universitario Insular de Gran Canaria, Las \\ Palmas de Gran Canaria, España
}

\begin{abstract}
Resumen
Objetivo: Análisis de los valores de hormona paratiroidea (PTH) intranodular obtenidos por punción con aguja fina como factor predictivo de hipoparatiroidismo posoperatorio. Método: De los 157 pacientes a los que se realizó la medición de la PTH intranodular, se estudiaron 37 que fueron intervenidos quirúrgicamente, estableciendo 15 variables para analizar: edad, sexo, tiempo de evolución, tamaño, cirugía de tiroides asociada, tratamiento preoperatorio con calcimiméticos, litiasis renal, densitometría ósea, aclaramiento de creatinina, calcio y PTH preoperatorios, PTH determinada tras punción con aguja fina, descenso de la PTH intraoperatoria, calcio posoperatorio y malignidad de la tumoración. Estas se compararon con la prueba de ji al cuadrado o el test exacto de Fisher, siendo significativa una $p<0.05$, construyéndose así un análisis de regresión logística. Resultados: El uso de calcimiméticos previo a la cirugía, la PTH preoperatoria > 88 pg/dl, el calcio preoperatorio $>12 \mathrm{mg} / \mathrm{dl}$, los valores de PTH intranodular > $2700 \mathrm{pg} / \mathrm{dl}$, el descenso de la PTH intraoperatoria a < 15 pg/dl y el calcio posoperatorio < 8,5 mg/dl fueron variables independientes significativas en el análisis univariante $(p<0.05)$ para la predicción del hipoparatiroidismo posoperatorio. Conclusiones: Los valores de PTH intranodular pueden predecir qué pacientes tienen mayor riesgo de sufrir un hipoparatiroidismo posoperatorio. Este factor debe tenerse en cuenta durante el estudio y el seguimiento de los pacientes para identificar precozmente posibles complicaciones.
\end{abstract}

PALABRAS CLAVE: Hormona paratiroidea. Hipoparatiroidismo posoperatorio. Calcio. Paratiroidectomía.

\begin{abstract}
Objective: To analize if parathyroid hormone (PTH) washout levels can be considered a predictive factor of postoperative hypoparathyroidism and determine its importance relative to other features already known as intraoperative PTH, Ca and PTH preoperative, and others. Method: Of the 157 patients to whom a fine needle aspiration (FNA) biopsy with intranodular parathyroid hormone level measurement was performed (washout PTH) 37 who underwent surgery were studied. 15 variables were taken into account when selecting patients for this study: age, gender, evolution length, size, associated thyroid surgery, preoperative treatment with calcimimetics, presence of renal lithiasis, bone densitometry alterations, creatine clearance, preoperative calcium and $P$ TH levels, $P$ TH scores obtained by fine needle aspiration, intraoperative $P$ TH decrease, postoperative calcium levels and tumour malignancy. We compare the nominal values, using the Chi squared or the exact Fisher test, where $p<0.05$ was considered statistically significant and perform a logistic regression analysis. Results: The use of calci-
\end{abstract}

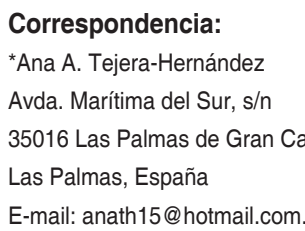

Fecha de aceptación: 14-05-2018

DOI: $10.24875 / \mathrm{CIRU} .18000026$
Cir Cir. 2018;86:475-480

Contents available at PubMed www.cirugiaycirujanos.com 
mimetics prior to surgery, the preoperative PTH level above $88 \mathrm{pg} / \mathrm{dl}$, the preoperative calcium above $12 \mathrm{mg} / \mathrm{dl}$, washout PTH above $2700 \mathrm{pg} / \mathrm{dl}$, intraoperative PTH level decrease below $15 \mathrm{pg} / \mathrm{dl}$, and postoperative calcium levels under $8.5 \mathrm{mg} / \mathrm{dl}$ were all independently significant variables in the univariant analysis $(p<0.05)$ in order to establish an early postoperative hypoparathyroidism. The rest of the variables were not statistically significative. Conclusion: The PTH washout levels can predict the appearance of postoperative hypoparathyroidism. This factor must be taken into account during the study and follow-up to identify possible complications and establish an adequate treatment on time.

KEY WORDS: Parathyroid hormone. Postoperative hypoparathyroidism. Calcium. Parathyroidectomy.

\section{Introducción}

Posterior a la intervención quirúrgica de un paciente con hiperparatiroidismo primario se produce una disminución rápida de los valores de calcio, debido a un desequilibrio reactivo entre la producción y la reabsorción ósea que produce un aumento de la captación en el hueso. La repercusión clínica de este mecanismo se traduce en la presencia de parestesias, trastornos neurológicos y alteraciones cardiacas ${ }^{1}$. La prevención del hipoparatiroidismo posoperatorio debe ser precoz, y además de preservar el resto de las glándulas paratiroideas hay que tener en cuenta ciertos factores que pueden predecir qué pacientes tienen mayor riesgo de sufrir esta alteración.

La medición de la PTH intranodular por punción con aguja fina es una técnica novedosa con beneficios y riesgos conocidos, que se utiliza para la localización y la diferenciación de la glándula paratiroidea de la tiroidea ${ }^{2-4}$. Estos valores asociados a otros factores podrían predecir el desarrollo de un hipoparatiroidismo posoperatorio, y ser una herramienta útil en la práctica clínica actual. En este estudio determinaremos su importancia en comparación con otras características ya conocidas, como la PTH intraoperatoria, y el calcio o la PTH preoperatorios, entre otros.

\section{Método}

Estudio retrospectivo observacional con muestreo consecutivo llevado a cabo durante los años 2007 y 2015 en el Hospital Universitario Insular de Gran Canaria, con 157 pacientes con diagnóstico de hiperparatiroidismo primario a los que se realizó la medición de los valores de PTH intranodular obtenidos por punción con aguja fina. Se estudiaron finalmente los 37 pacientes que fueron intervenidos en este periodo, con un seguimiento posoperatorio mínimo de 12 meses que concluyó en enero de 2017, identificando quiénes habían presentado hipoparatiroidismo posoperatorio y que factores influyeron en su aparición.

Se incluyeron en el estudio todos los pacientes intervenidos con diagnóstico de hiperparatiroidismo primario a los que se había realizado previamente la medición de la PTH intranodular, y solo se hubiese resecado una glándula, y se excluyó a los pacientes con enfermedad multiglandula, con cirugías previas en otra de las glándulas, con cirugía de autotrasplante y que no cumplieran con la recogida de datos de todas las variables a estudiar.

Se establecieron y estudiaron 15 variables: edad, sexo, tiempo de evolución de la enfermedad, tamaño, cirugía de tiroides asociada, tratamiento preoperatorio con calcimiméticos, presencia de litiasis renal, alteraciones en la densitometría ósea (T-score $<2.5$ ), aclaramiento de creatinina $(<60 \mathrm{ml} / \mathrm{min})$, calcio y PTH preoperatorios, valores de PTH obtenidos por punción con aguja fina, descenso de la PTH intraoperatoria (10 minutos tras la escisión), calcio posoperatorio (6 horas tras la escisión) y malignidad de la tumoración.

Se categorizaron las variables cuantitativas para transformarlas en nominales con dos grupos homogéneos que fueran más comparables, lo cual, además, evitó la dispersión de la muestra. En cuanto a la edad, se eligió un punto de corte de 50 años porque es uno de los criterios de intervención quirúrgica en pacientes asintomáticos, y la edad menor que esta se relaciona con mayor riesgo de malignidad. Los nódulos de gran tamaño y un largo tiempo de evolución de la enfermedad son variables asociadas a un aumento de la aparición de hipoparatiroidismo posoperatorio, por lo decidimos una medida de corte de gran tamaño $(2 \mathrm{~cm})$ y con mayor tiempo de espera para la cirugía (2 años). Para decidir una actitud quirúrgica en los pacientes asintomáticos con hiperparatiroidismo primario existen valores definidos en la densitometría ósea y el aclaramiento de creatinina, los cuales se utilizaron para establecer el punto de corte de estas variables. Con respecto a la PTH, los valores normales en nuestro laboratorio son de 15 a 88 pg/dl, 
por lo que decidimos estudiar esta última cifra como factor predictivo. El calcio corregido por la albúmina en la normalidad se encuentra entre 8.5 y $10.5 \mathrm{mg} / \mathrm{dl}$, considerando un aumento franco de $1.5 \mathrm{mg} / \mathrm{dl}$ por encima del valor alto de normalidad y definiendo así los $12 \mathrm{mg} / \mathrm{dl}$ descritos en nuestra variable de estudio. El descenso de la PTH intraoperatoria por debajo de $15 \mathrm{pg} / \mathrm{dl}$ y el descenso del calcio posoperatorio por debajo de $8.5 \mathrm{mg} / \mathrm{dl}$ son factores predictores conocidos ${ }^{5}$, por lo que también decidimos estudiarlos.

Los valores de PTH intranodular obtenidos por punción con aguja fina son patológicos cuando están por encima de los $100 \mathrm{pg} / \mathrm{dl}$, pero nunca se habían establecido como variable predictora de hipoparatiroidismo, por lo que decidimos construir múltiples análisis univariantes hasta obtener un valor de corte significativo en $2700 \mathrm{pg} / \mathrm{dl}$.

Con el fin de comparar las variables nominales se crearon tablas de contingencia para emplear la prueba de ji al cuadrado o el test exacto de Fisher, siendo significativa una $p<0.05$. Al determinar las variables que tenían relación significativa con la presencia de hipoparatiroidismo posoperatorio, de forma independiente se inició la elaboración de múltiples modelos de análisis multivariante, construyendo así un análisis de regresión logística utilizando el programa IBM® SPSS $^{\circledR}$ Statistics V. 21.

\section{Resultados}

Se realizaron 157 mediciones de los valores de PTH intranodular mediante punción con aguja fina guiada por ecografía. Se intervino quirúrgicamente a 37 pacientes, de los cuales 17 (46\%) presentaron posteriormente hipoparatiroidismo posoperatorio, siendo transitorio en todos los casos $(<12$ meses de evolución) y sintomático solo en $4(24 \%)$, con leves parestesias y calambres musculares, que se solucionaron solo con calcio y vitamina $D$ oral.

El uso de calcimiméticos previo a la cirugía, la PTH preoperatoria $>88 \mathrm{pg} / \mathrm{dl}$, el calcio preoperatorio $>12 \mathrm{mg} / \mathrm{dl}$, la PTH intranodular > $2700 \mathrm{pg} / \mathrm{dl}$, el descenso de la PTH intraoperatoria $<15 \mathrm{pg} / \mathrm{dl}$ y el calcio posoperatorio $<8,5 \mathrm{mg} / \mathrm{dl}$ fueron variables independientes significativas en el análisis univariante $(p<0.05)$ para la predicción de hipoparatiroidismo posoperatorio (Tabla 1); el resto de las variables no fueron estadísticamente significativas.

El análisis multivariable reveló que el uso de calcimiméticos previo a la cirugía, el descenso de la PTH
Tabla 1. Características de los pacientes intervenidos

\begin{tabular}{|c|c|c|c|}
\hline \multirow[t]{2}{*}{ Característica } & \multicolumn{2}{|c|}{$\begin{array}{l}\text { Hipoparatiroidismo } \\
\text { posoperatorio }\end{array}$} & \multirow[t]{2}{*}{$p$} \\
\hline & $\begin{array}{c}\text { Presente } \\
\text { n (\%) }\end{array}$ & $\begin{array}{c}\text { Ausente } \\
\mathrm{n}(\%)\end{array}$ & \\
\hline Número de casos & $17(46)$ & $20(54)$ & \\
\hline $\begin{array}{l}\text { Edad (años) } \\
\quad \leq 50 \\
>50\end{array}$ & $\begin{array}{l}9(53) \\
8(47)\end{array}$ & $\begin{array}{c}7(35) \\
13(65)\end{array}$ & $<0.272$ \\
\hline $\begin{array}{l}\text { Sexo } \\
\text { Mujer } \\
\text { Varón }\end{array}$ & $\begin{array}{l}12(73) \\
5(27)\end{array}$ & $\begin{array}{l}13(65) \\
7(35)\end{array}$ & $<0.717$ \\
\hline $\begin{array}{l}\text { Tiempo de evolución (años) } \\
\leq 2 \\
>2\end{array}$ & $\begin{array}{c}5(27) \\
12(73)\end{array}$ & $\begin{array}{c}6(30) \\
14(70)\end{array}$ & $<0.968$ \\
\hline $\begin{array}{l}\text { Tamaño tumoral }(\mathrm{mm}) \\
\quad<20 \\
\geq 20\end{array}$ & $\begin{array}{l}11(65) \\
6(35)\end{array}$ & $\begin{array}{l}17(85) \\
3(15)\end{array}$ & $<0.151$ \\
\hline $\begin{array}{l}\text { Cirugía de tiroides asociada } \\
\text { Sí } \\
\text { No }\end{array}$ & $\begin{array}{c}5(27) \\
12(73)\end{array}$ & $\begin{array}{c}5(25) \\
15(75)\end{array}$ & $<0.763$ \\
\hline $\begin{array}{l}\text { Tratamiento calcimimético } \\
\text { Sí } \\
\text { No }\end{array}$ & $\begin{array}{l}12(73) \\
5(27)\end{array}$ & $\begin{array}{c}4(20) \\
16(80)\end{array}$ & $<0.001$ \\
\hline $\begin{array}{l}\text { Litiasis renal } \\
\text { Sí } \\
\text { No }\end{array}$ & $\begin{array}{c}5(27) \\
12(73)\end{array}$ & $\begin{array}{c}5(25) \\
15(75)\end{array}$ & $<0.763$ \\
\hline $\begin{array}{l}\text { Densidad mineral ósea, T-score } \\
\quad<2.5 \\
>2.5\end{array}$ & $\begin{array}{c}7(41) \\
10(59)\end{array}$ & $\begin{array}{c}6(30) \\
14(70)\end{array}$ & $<0.477$ \\
\hline $\begin{array}{l}\text { Aclaramiento de creatinina }(\mathrm{ml} / \mathrm{min}) \\
\geq 60 \\
<60\end{array}$ & $\begin{array}{l}14(82) \\
3(18)\end{array}$ & $\begin{array}{l}17(85) \\
3(15)\end{array}$ & $<0.827$ \\
\hline $\begin{array}{l}\text { PTH preoperatoria }(\mathrm{pg} / \mathrm{dl}) \\
\geq 88 \\
<88\end{array}$ & $\begin{array}{l}11(65) \\
6(35)\end{array}$ & $\begin{array}{c}4(20) \\
16(80)\end{array}$ & $<0.005$ \\
\hline $\begin{array}{l}\text { Calcio preoperatorio }(\mathrm{mg} / \mathrm{dl}) \\
\geq 12 \\
<12\end{array}$ & $\begin{array}{c}7(41) \\
10(59)\end{array}$ & $\begin{array}{l}2(10) \\
18(90)\end{array}$ & $<0.027$ \\
\hline $\begin{array}{l}\text { PTH intranodular (pg/dl) } \\
\quad \geq 2700 \\
\quad<2700\end{array}$ & $\begin{array}{c}7(41) \\
10(59)\end{array}$ & $\begin{array}{c}1(5) \\
19(95)\end{array}$ & $<0.007$ \\
\hline $\begin{array}{l}\text { PTH intraoperatoria }(\mathrm{pg} / \mathrm{dl}) \\
\quad>15 \\
\leq 15\end{array}$ & $\begin{array}{l}10(59) \\
7(41)\end{array}$ & $\begin{array}{c}19(95) \\
1(5)\end{array}$ & $<0.007$ \\
\hline $\begin{array}{l}\text { Calcio posoperatorio }(\mathrm{mg} / \mathrm{dl}) \\
>8.5 \\
\leq 8.5\end{array}$ & $\begin{array}{c}13(76) \\
4(24)\end{array}$ & $\begin{array}{c}20(100) \\
0(0)\end{array}$ & $<0.021$ \\
\hline $\begin{array}{l}\text { Malignidad } \\
\text { Sí } \\
\text { No }\end{array}$ & $\begin{array}{c}3(18) \\
14(82)\end{array}$ & $\begin{array}{c}1(5) \\
19(95)\end{array}$ & $<0.216$ \\
\hline
\end{tabular}

PTH: hormona paratiroidea. 
Tabla 2. Análisis de regresión logística

\begin{tabular}{lcc}
\hline Variable independiente & OR (IC 95\%) & p \\
\hline Tratamiento calcimimético & $4,67(0,288-0,734)$ & $<0.001$ \\
PTH intranodular $\geq 2700 \mathrm{pg} / \mathrm{dl}$ & $2,191(0,023-0,667)$ & $<0.036$ \\
PTH intraoperatoria $\leq 15 \mathrm{pg} / \mathrm{dl}$ & $3,174(0,152-0,702)$ & $<0.003$ \\
PTH preoperatoria $\geq 88 \mathrm{pg} / \mathrm{dl}$ & & $<0.585$ \\
Calcio preoperatorio $\geq 12 \mathrm{mg} / \mathrm{dl}$ & & $<0.218$ \\
Calcio posoperatorio $\leq 8.5 \mathrm{mg} / \mathrm{dl}$ & & $<0.412$ \\
\hline IC: intervalo de confianza; OR: odds ratio. & &
\end{tabular}

intraoperatoria $<15 \mathrm{pg} / \mathrm{dl}$ y los valores de PTH intranodular $>2700 \mathrm{pg} / \mathrm{dl}$ son factores que aumentan el riesgo de presentar hipoparatiroidismo posoperatorio (Tabla 2).

\section{Discusión}

El hipoparatiroidismo posoperatorio es la complicación más frecuente que se presenta en el seguimiento clínico de los pacientes tras una cirugía paratiroidea, y se define como un descenso de la PTH y la aparición de manifestaciones clínicas relacionadas con el descenso del calcio, como parestesias, disestesias periorales, espasmos musculares, mioclonías, debilidad, cefalea y náuseas, que incluso pueden poner en peligro la vida del paciente ${ }^{5,6}$. Cuando se presenta en pacientes intervenidos por hiperparatiroidismo primario, suelen estar relacionados con la hiperfunción previa de la glándula, el mal funcionamiento del resto de las glándulas, las alteraciones en la producción y liberación de PTH, la manipulación quirúrgica, el síndrome del hueso hambriento y las características propias tanto de la tumoración como del paciente ${ }^{5-8}$. El porcentaje de aparición de esta alteración varía según los autores, pero se encuentra entre un 10 y un $30 \%$ de los $\operatorname{casos}^{5,9,10}$, siendo en nuestro servicio del $46 \%$, lo cual puede explicarse porque nuestro hospital concentra su actividad quirúrgica en enfermedades oncológicas, interviniendo por hiperparatiroidismo primario solo a pacientes con patologías asociadas, casos complicados, de mayor dificultad técnica quirúrgica, con sospecha de malignidad y con riesgo anestésico elevado, teniendo que esperar de acuerdo a la disponibilidad para ser intervenidos, aumentando así el uso de tratamientos previos, con mayor tiempo de hiperfuncionamiento de la glándula afecta. En el estudio se realizó un seguimiento mínimo de 12 meses y no hubo casos de hipoparatiroidismo permanente, ya que solo estudiamos pacientes con cirugías de una glándula manteniendo el resto preservadas.
Existen muchas características que pueden aumentar el riesgo de tener hipoparatiroidismo posoperatorio, y aunque este trabajo busca las ventajas del uso de la medición de la PTH intranodular, debíamos estudiar además el resto de los posibles factores para comparar su utilidad.

La edad menor de 50 años es un factor a tener en cuenta para decidir una actitud quirúrgica en los pacientes con hiperparatiroidismo asintomático, pues se relaciona con un mayor riesgo de malignidad y un mayor tiempo de exposición a la enfermedad; al estudiarla no encontramos una relación con la aparición posterior de hipoparatiroidismo ${ }^{11}$. El aclaramiento de creatinina $<60 \mathrm{ml} / \mathrm{min}$ y un $T$-score $<2.5$ en la densitometría ósea son parámetros que deciden la intervención quirúrgica de un paciente asintomático y están relacionados con las alteraciones atribuidas a un hiperparatiroidismo primario mal controlado; además, las alteraciones prolongadas en el tiempo del recambio óseo llevan a la producción posterior de un síndrome de hueso hambriento con una mayor captación del calcio, lo que conlleva una hipocalcemia descontrolada ${ }^{11-13}$. A pesar de esto, en nuestro estudio estas variables no fueron significativas.

Cuando se realiza una exploración tiroidea en el mismo acto quirúrgico de la exéresis de una tumoración paratiroidea existe el riesgo, durante la movilización, de producir la exéresis, la desvascularización o un traumatismo del resto de las glándulas, lo que aumentaría el riesgo de un hipoparatiroidismo posterior $^{14-16}$; por ello, decidimos estudiar esta variable, que no fue relevante en nuestros pacientes. La mayor cantidad de casos de hiperparatiroidismo se presentó en mujeres, al igual que en otros estudios ${ }^{5,8,10}$, pero ello no estuvo en relación con la aparición posterior de hipoparatiroidismo. Los valores altos de PTH durante largo tiempo, así como el aumento de su producción en el caso de adenomas o carcinomas de gran tamaño, producen una adaptación de los receptores del calcio a una hipercalcemia continua que se suprime bruscamente durante la cirugía, lo que conlleva, en la mayoría de los pacientes, una hipocalcemia reactiva a este mecanismo ${ }^{5,17}$; sin embargo, a pesar de que estas variables son importantes en la práctica clínica, en nuestro estudio no resultaron significativas.

Los calcimiméticos actúan sobre el receptor sensible al calcio situado en la superficie de la célula principal de la glándula paratiroides incrementando su sensibilidad, lo que reduce las concentraciones de PTH. Esta reducción se asocia a un descenso 
paralelo de las concentraciones séricas de calcio ${ }^{18,19}$. En nuestro hospital utilizamos estos fármacos siguiendo los criterios de las guías de la Agencia Europea de Medicamentos ${ }^{20}$ para el tratamiento del hiperparatiroidismo secundario en pacientes con insuficiencia renal crónica en diálisis y para la reducción de la hipercalcemia en pacientes con carcinoma de paratiroides o hiperparatiroidismo primario, para los que, según sus valores de calcio sérico, estaría indicada la paratiroidectomía, sobre todo cuando hay que esperar mucho para un tratamiento quirúrgico, los valores de calcio son muy elevados o el paciente presenta síntomas importantes de hipercalcemia, suspendiéndose 24 horas antes de la cirugía. Aunque su vida media es de 6 horas, pueden llegar a acumularse en los tejidos y mantener sus efectos hasta 40 horas después de su suspensión, lo que podría explicar su relación significativa con la presencia de hipoparatiroidismo posoperatorio en nuestros pacientes ${ }^{18-20}$. Teniendo en cuenta estos resultados, se plantea la necesidad de suspender el fármaco al menos 2 días antes de la intervención quirúrgica.

Los valores de PTH y de calcio preoperatorios elevados son factores ampliamente estudiados que se relacionan con la presencia de hipoparatiroidismo posoperatorio ${ }^{5}$, lo cual confirmamos con nuestro estudio. Los valores de descenso de la PTH intraoperatoria siguen siendo controvertidos, obteniendo en algunos estudios una relación significativa con valores inferiores a $10 \mathrm{pg} / \mathrm{dl}$ y en otros por debajo de $15 \mathrm{pg} / \mathrm{dl}$, e incluso se han conseguido resultados predictivos cuando el descenso es mayor de un $60 \%$ del valor inicial de PTH ${ }^{21-24}$. En nuestro estudio, fue el descenso de los valores por debajo de $15 \mathrm{pg} / \mathrm{dl}$ el que demostró una relación significativa con la aparición posterior de hipoparatiroidismo.

Tras la exéresis de la glándula patológica, el calcio puede tardar en descender de 24 a 72 horas, por lo que valores por debajo de lo normal a las 6 horas de la escisión debería ser un dato de alarma que debe tenerse en cuenta para prevenir una probable hipocalcemia ${ }^{25}$; esta relación fue significativa en nuestro estudio.

La medición de los valores de PTH intranodular obtenidos por punción con aguja fina guiada por ecografía se utiliza principalmente para localizar la glándula afecta y confirmar el origen paratiroideo de la tumoración. Estos valores son variables, pero cuando se encuentran por encima de los $100 \mathrm{pg} / \mathrm{dl}$ se consideran patológicos ${ }^{26,27}$. Realizando múltiples análisis univariantes con las cifras obtenidas en nuestras diferentes punciones, obtuvimos que los valores por encima de $2700 \mathrm{pg} / \mathrm{dl}$ tenían una relación significativa con la aparición de hipoparatiroidismo posterior, tanto en el análisis univariante como en el multivariante. Probablemente esta relación se explique por la excesiva hiperfunción de la glándula, con una alta producción de PTH, a la cual el organismo se adapta y al ser suprimida con la resección quirúrgica se produce una hipocalcemia reactiva. Los resultados de esta medición deben ser tomados con cautela, ya que se trata de una muestra pequeña y no existe ningún otro estudio que haya buscado dicha relación, lo que dificulta la comparación de nuestros resultados con la literatura actual.

La medición de los valores de PTH podría ser un factor a tener en cuenta en el futuro, pues asociado con el resto de las variables conocidas podría predecir la aparición de un hipoparatiroidismo posoperatorio, y permitir el establecimiento de un diagnóstico y de un tratamiento precoz que disminuiría las manifestaciones clínicas desfavorables para estos pacientes.

\section{Conclusiones}

El uso de calcimiméticos previo a la cirugía, la elevación de los valores de PTH y de calcio preoperatorios, el descenso de la PTH intraoperatoria y los bajos valores de calcio posoperatorio son variables conocidas que, junto con la medición de los valores de PTH intranodular, pueden predecir qué pacientes tienen mayor riesgo de sufrir hipoparatiroidismo posoperatorio, siendo una herramienta útil en la práctica clínica actual para obtener mejores resultados y evitar complicaciones que prolonguen la estancia hospitalaria y disminuyan la calidad de vida de nuestros pacientes.

\section{Conflicto de intereses}

Los autores de este trabajo declaran no tener ningún conflicto de intereses.

\section{Bibliografía}

1. Vélayoudom-Céphise FL, Wémeau JL. Primary hyperparathyroidism and vitamin D deficiency. Ann Endocrinol. 2015;76:153-62.

2. Bancos I, Grant C, Nadeem S, Stan M, Reading C, Sebo T, et al. Risks and benefits of parathyroid fine-needle aspiration with parathyroid hormone washout. Endocr Pract. 2012;18:441-9.

3. Ketha H, Lasho M, Algeciras-Schimnich A. Analytical and clinical validation of parathyroid hormone (PTH) measurement in fine-needle aspiration biopsy (FNAB) washings. Clin Biochem. 2016;49:16-21.

4. Giusti M, Dolcino M, Vera L, Ghiara C, Massaro F, Fazzuoli L, et al. Institutional experience of PTH evaluation on fine-needle washing after aspiration biopsy to locate hyperfunctioning parathyroid tissue. J Zhejiang Univ Sci B. 2009;10:323-30. 
5. Stack B, Bimston D, Bodenner D, Brett E, Dralle H, Orloff L, et al. American Association of Clinical Endocrinologists and American College of Endocrinology Disease state clinical review: postoperative hypoparathyroidism - definitions and management. Endocr Pract. 2015; 21:674-85.

6. Chen Y, Masiakos P, Gaz R, Hodin R, Parangi S, Randolph G, et al. Pediatric thyroidectomy in a high volume thyroid surgery center: risk factors for postoperative hypocalcemia. J Pediatr Surg. 2015;50:1316-9.

7. Lorente-Poch L, Sancho J, Ruiz S, Sitges-Serra A. Importance of in situ preservation of parathyroid glands during total thyroidectomy. $\mathrm{Br} J$ Surg. 2015;102:359-67.

8. Cusano N, Anderson L, Rubin M, Silva B, Costa A, Irani D, et al. Recovery of parathyroid hormone secretion and function in postoperative hypoparathyroidism: a case series. J Clin Endocrinol Metab. 2013 98:4285-90.

9. American Thyroid Association Surgery Working Group; American Association of Endocrine Surgeons,; American Academy of Otolaryngology-Head and Neck Surgery; American Head and Neck Society, Carty SE, Cooper DS, Doherty GM, Duh QY, Kloos RT, Mandel SJ, et al. Consensus statement on the terminology and classification of central neck dissection for thyroid cancer. Thyroid. 2009;19:1153-8.

10. Walker V, Jan De Beur S. Postoperative hypoparathyroidism: medical and surgical therapeutic options. Thyroid. 2009;19:967-73.

11. Udelsman R, Åkerström G, Biagini C, Duh QY, Miccoli P, Niederle B, et al. The surgical management of asymptomatic primary hyperparathyroidism: proceedings of the Fourth International Workshop. J Clin Endocrinol Metab. 2014;99:3595-606.

12. Underbjerg L, Sikjaer T, Mosekilde L, Rejnmark L. Postsurgical hypoparathyroidism - risk of fractures, psychiatric diseases, cancer, cataract, and infections. J Bone Miner Res. 2014;29:2504-10.

13. Moure MD, Luque-Ramírez M, López G, López M, Gómez-Pan A. Síndrome del hueso hambriento relacionado con hipertiroidismo. An Med Intern. 2006;23:326-8.

14. Rajaei MH, Oltmann SC, Schneider DF, Sippel RS, Chen H. Outcomes after subtotal parathyroidectomy for primary hyperparathyroidism due to hyperplasia: significance of whole vs. partial gland remnant. Ann Surg Oncol. 2015;22:966-71.

15. Merchavy S, Marom T, Forest VI, Hier M, Mlynarek A, McHugh T, et al. Comparison of the incidence of postoperative hypocalcemia following total thyroidectomy vs completion thyroidectomy. Otolaryngol Head Neck Surg. 2015;152:53-6.
16. Hasse C, Sitter H, Brune M, Wollenteit I, Nies C, Rothmund M. Quality of life and patient satisfaction after reoperation for primary hyperparathyroidism: analysis of long-term results. World J Surg. 2002;26:1029-36.

17. Sillero A, Atienza MA. Diagnostic-therapeutic management of parathyroid carcinoma. An Med Intern. 2002;19:644-8.

18. Torun D, Yildiz I, Micozkadioglu H, Nursal GN, Yigit F, Ozelsancak R. The effects of cinacalcet treatment on bone mineral metabolism, anemia parameters, left ventricular mass index and parathyroid gland volume in hemodialysis patients with severe secondary hyperparathyroidism. Saudi J Kidney Dis Transpl. 2016;27:15-22.

19. Mingione A, Verdelli C, Terranegra A, Soldati L, Corbetta S. Molecular and clinical aspects of the target therapy with the calcimimetic cinacalcet in the treatment of parathyroid tumors. Curr Cancer Drug Targets. 2015;15:563-74.

20. Mercadal G, Blasco I. Retrospective assessment of effective and safety of cinacalcet for the treatment of secondary hyperparathyroidism depending on basal iPTH level. Farm Hosp. 2012;36:11-5.

21. Wang J, Gu J, Han Q, Wang W, Shang J. Value of intraoperative parathyroid hormone monitoring in papillary thyroid cancer surgery: can it be used to guide the choice of operation methods? Int J Clin Exp Med. 2015;8:7778-85

22. Rivere AE, Brooks AJ, Hayek GA, Wang H, Corsetti RL, Fuhrman GM. Parathyroid hormone levels predict posttotal thyroidectomy hypoparathyroidism. Am Surg. 2014;80:817-20.

23. Nilubol N, Weisbrod AB, Weinstein LS, Simonds WF, Jensen RT, Phan GQ, et al. Utility of intraoperative parathyroid hormone monitoring in patients with multiple endocrine neoplasia type 1-associated primary hyperparathyroidism undergoing initial parathyroidectomy. World $\mathrm{J}$ Surg. 2013;37:1966-72.

24. Roshan A, Kamath B, Roberts S, Atkin SL, England RJ. Intra-operative parathyroid hormone monitoring in secondary hyperparathyroidism: is it useful? Clin Otolaryngol. 2006;31:198-203.

25. Rosa KM, Matos LL, Cernea CR, Brandão LG, Araújo VJ. Postoperative calcium levels as a diagnostic measure for hypoparathyroidism after total thyroidectomy. Arch Endocrinol Metab. 2015;21:30.

26. Abdelghani R, Noureldine S, Abbas A, Moroz K, Kandil E. The diagnostic value of parathyroid hormone washout after fine-needle aspiration of suspicious cervical lesions in patients with hyperparathyroidism. Laryngoscope. 2013;123:1310-3.

27. Ing SW, Pelliteri PK. Diagnostic fine-needle aspiration biopsy of an intrathyroidal parathyroid gland and subsequent eucalcemia in a patient with primary hyperparathyroidism. Endocr Pract. 2008;14:80-6. 